(Aus dem physiologischen Institut der Universität Lemberg.)

\title{
Zur Lehre Munk's über Beginn und Reihen- folge in der Ausbreitung der Bewegungen bei Rückenmarksreflexen, wie bei Tätigkeit der sogenannten „Prinzipalzentren“" 1).
}

Von

Prof. Dr. Adolf Beck und Prof. Dr. Gustav Bikeles.

(Nit 2 Textflguren.)

Es bleibt ein ungeschmälertes Verdienst Munk's, dass er trotz der bekannten Versuche Goltz's, die der Grosshirnrinde jedwede Rolle für die Motilität zu benehmen schienen, die grosse Bedeutung der psychomotorischen Region (oder der von $\mathrm{Munk}$ so benannten Fühlsphäre) scharf betonte. In einer grossen Reihe von Arbeiten war $\mathrm{Munk}^{2}$ ) bestrebt - und zwar mit grossem Erfolg - darzutun, dass nach Exstirpation der Extremitätenregion der Hirnrinde beim Tier die nachher ausführbaren Bewegungen auf der kontralateralen Seite Gemeinschaftsbewegungen sind (Gehen, Laufen, beim Affen auch Klettern usw.), während feinere, vereinzelte Bewegungen dem so operierten Tiere auf dieser Seite hleibend abgehen.

Munk sprach demgemäss in seinen ersten Arbeiten von (erhaltenen) Gemeinschaftsbewegungen, d. h. „Bewegungen, welche an den gegenseitigen Extremitäten zusammen in Verbindung oder in der Reihe mit Bewegungen anderer Körperteile erfolgen", und (verlorengegangenen) Sonderbewegungen. In den nachfolgenden Publikationen ${ }^{3}$ ) ersetzte Munk den Ausdruck „Gemeinschaftsbewegung."

1) Vorgelegt der Krakauer Akademie der Wissenschaften in der Sitzung rom 10. Oktober 1910. Erscheint polnisch in den Abhandl. dieser Akad. Bd. 10 B.

2) Munk, Über die Fühlsphären der Hirnrinde. Sitzungsber. d. preuss. Akad. d. Wissensch. 1892. - Über die Funktionen von Hirn und Rückenmark. Berlin 1909.

3) Munk, Weitere Mitteilungen $1898 \mathrm{ff}$. 
vielleicht überflüssigerweise durch "Prinzipalbewegung" und spricht auch von Prinzipalzentren, „welche unterhalb der Grosshirnrinde zwischen dieser und dem Rückenmarke gelegen sind"

Von diesen supponierten Prinzipalzentren sollen nun nach $\mathrm{Munk}$ zu den motorischen Rückenmarkszentren der Extremitäten beson dere, von den kortikospinalen Bahnen verschiedene Leitungsbahnen zieben. Dieselben unterscheiden sich nach $\mathrm{Munk}$ in ihrer Verbindungsweise mit den die Bewegungen in den einzelnen Gelenken auslösenden Rückenmarkszentren wesentlich von der Verbindungsart der Leitungsbahnen der Extremitätenregion der Hirnrinde (mit diesen Zentren).

Die Leitungsbahnen der Extremitätenregion sollen wohl mit jedem einzelnen Zentrum für die Motilität je eines Gelenkes Verbindungen haben. Hingegen meint $\mathrm{Munk}$, dass die Leitungsbahnen der Prinzipalzentren nicht auf jedes einzelne dieser Rückenmarkszentra einwirken, sondern zunächst und unmittelbar nur auf dasjenige der obersten Gelenke, vielleicht richtiger des obersten Gelenkes ${ }^{1}$ ).

Auf diese Weise will Munk eine ausreichende Erklärung dafür gefunden haben, dass nach der Exstirpation der Extremitätenregion von ihm Sonderbewegungen namentlich der untersten Gelenke vermisst wurden. Die direkte Verbindung nämlich der Prinzipalzentren durch ihre zum Rückenmark verlaufenden Leitungsbahnen ausschliesslich mit dem Rückenmarkszentrum, welches die Motilität des obersten Gliedes vermittelt, verschuldet es, dass nunmehr nur ein typisch ablaufender Innervationsvorgang noch möglich ist, nämlich rom obersten Gliede der Extremität zum untersten fortschreitend.

Ganz analog wie die Erregung der motorischen Rückenmarkszentren von den Prinzipalzentren aus in bezug auf Angriffsort und Ausbreitungsart sich abspielt, soll auch nach Munk die im Rückenmark bei Reizung an der Peripherie erzeugte reflektorische Erregung: (Rückenmarksreflexe) sich verhalten. Das heisst auch in diesem Falle betrifft nach Munk die Erregung zunächst das Zentrum für das oberste Gelenk, und bei Steigerung der Reizung schreitet die Er-

1) So sagt Munk ausdrücklich: „Für sich allein kann bloss das oberste Glied tätig werden, jedes andere Glied ist mit seiner Tätigkeit an die Tätigkeit der dem Rumpfe näheren Ftlieder gebunden"; weiter unten wieder "welche durch Leitungsbahnen in derselben Reihenfolge miteinander verbunden sind, wie die Glieder sich aneinander schliessen". (Über die Funktionen von Hirn und Rückenmark. S. 63.) 
regung in der Richtung vom Zentrum für das oberste $\mathrm{zu}$ dem für die untersten Gelenke fort.

Behufs Erklärung des letzteren Verhaltens im Rückenmark supponiert Munk weiter ganz bypothetisch, dass die ins Rückenmark eintretenden zentripetalen (sensiblen) Nervenfasern nicht gleichmässig auf die einzelnen Rückenmarkszentra für jedes Gelenk einwirken, sondern sei es direkt, sei es indirekt durch Zellen zunächst die etwaige Frregung dem Zentrum der obersten Gelenke zuleiten, während die Zentra für die unteren Glieder ihre Erregung erst von dem Zentrum des obersten Gliedes also mittelbar und in bestimmter Ordnung - in der Reihenfolge der Gelenke - erhalten können.

Angesichts der hohen Berleutung der Untersuchungen Munk's für die Physiologie des Zentralnervensystems wie auch in Anbetracht dessen, dass die Arschaungen dieses Forschers in der Neurologie sehr leicht Anklang finden, stellten wir uns, da uns eine derartige Auffassungsweise auf Grund anderseitiger Untersuchungen nicht plausibel erschien, die Aufgabe, die obigen Hypothesen Munk's auf ihre Haltbarkeit zu prüfen.

Wir stellten uns dabei folgende spezielle Fragen:

1. Erfolgt denn wirklich nicht nach Exstirpation der Extremitätenregion der Hirnrinde, eventuell sogar nach Rückenmarksdurchschneidung eine vereinzelte Bewegung im untersten Gelenke?

2. Gibt es überhaupt, sei es nach Exstirpation der Extremitätenregion der Hirnrinde, sei es auch nach Rückenmarksdurchschneidung eine bestimmte und unabänderliche Reihenfolge in den Bewegungen der einzelnen Glieder der Extremitäten, welche auf eine Ausbreitung der Erregung in den Rückenmarkzentren im Sinne Munk's schliessen lässt?

3. Zeigt überhaupt irgendein motorisches Rückenmarkszentrum für die Bewegung in einem Gelenke eine deutlichere Anspruchsfähigkeit auf Erregung im Verhältnis zu den Zentren für die Bewegung in anderen Gelenken, und wenn, was mag die Ursache eines solchen Verhaltens sein?

Zur Beantwortung dieser Fragen dienten uns Tiere (Hunde) a) nach einseitiger Exstirpation der Extremitätenregion der Hirnrinde, b) nach Durchschneidung des Rückenmarks im Dorsalabschnitt und e) in Narkose.

Behufs Untersuchung wurden die so behandelten Tiere in eine Hängematte gebracht, aus welcher die Extremitäten durch ent- 
sprechende Einschnitte bequem und frei herunterhingen. Ein Teil der Tiere wurde auch in einem $\mathrm{zu}$ diesem $\mathrm{Z}$ wecke konstruierten breiten, mit Riemen zum Aufhängen versehenen, von innen ausgepolsterten Ledergürtel untersucht, wobei den Tieren nach Belieben eine vertikale oder eine horizontale Lage erteilt werden konnte.

Die angewandten Reize waren vor allem thermische, dann elektrische und auch mechanische.

Zum Zwecke der thermischen Reizung wurden weite und entsprechend tiefe Glasgefässe mit Wasser von einer Temperatur von etwa $55^{\circ}$ bis $60^{\circ} \mathrm{C}$ gefüllt und hierauf unter die zu untersuchende Extremität geschoben, so dass ein immer ungefähr gleicher Teil der Pfote in das Wasser eintauchte. In der Regel wurde das mit Wasser gefüllte Gefäss so lange hingehalten, bis das Tier die Extremität aus dem Wasser hervorzog. Sowohl der Beginn der Reizung, wie auch der Moment des Eintretens jeder durch diesen Reiz hervorgerufenen besonderen Bewegung der beobachteten Extremität wurde auf rotierender Trommel vermittels eines elektrischen Signals registriert. Dabei wurde anch die Art jeder einzelnen Bewegung genau beobachtet und notiert.

Es zeigte sich nun, dass das Zurückziehen der Pfote aus dem heissen Wasser bei verschiedenen Tieren und sogar bei demselben Tiere nicht immer einförmig oder durch Bewegung in einem und demselben Gelenke erfolgt. Manchmal war mit der ersten nach einiger Reizdauer ausgeführten Bewegung (Beugung) die Zurückziehung der Extremität aus dem heissen Wasser vollbracht. Sehr bäufig erfolgte nach Ablauf eines kürzeren oder längeren latenten Reizstadiums eine Bewegung (Beugung) in einem Gelenke, ohne dass es zum definitiven Zurückziehen der Extremität aus dem beissen Wasser kam. Nach einem kleineren oder grösseren Intervall, welches, wie oben angegeben, auf der Trommel signalisiert wurde, sah man dann eine abermalige Bewegung oder auch mehrere durch weitere Intervalle getrennte neuerliche Bewegungen. Dieselben betrafen sei es das schon anfangs aktiv gewesene Gelenk, sei es dieses und benachbarte Gelenke, sei es ausschliesslich ein oder mehrere benachbarte Gelenke.

Auf diese Weise erhielten wir häufig eine wahre Reihenfolge von sukzessiven Innervationsvorgängen in mebreren Gelenken, was gerade für die Beantwortung der oben gestellten Fragen von Bedeutung ist. 
Die Ergebnisse dieser Untersuchungen veranschaulichen die beigefügten Tabellen I bis V.

Zur Erläuterung sei angegeben, dass in allen nachfolgenden Tabellen bedeutet: $\mathrm{Sp} .=$ Sprungelenk, Kn. $=$ Kniegelenk, Hf. $=$ Hüftgelenk, Hnd. = Handgelenk, El. = Ellbogengelenk, Sch. = Schultergelenk.

Das Zeichen + bedeutet gleichzeitige Reaktion zweier oder auch mehrerer Gelenke; das Zeichen $\rightarrow$ das Auftreten einer abermaligen Reaktion nach einem Intervall. So bedeutet z. B. Sp. $\rightarrow$ Kn. Dorsalflexion im Sprunggelenk und hierauf, nach einem Zeitintervall von einem Bruchteil einer Sekunde bis einigen Sekunden, Beugung im Kniegelenk. Sp. $+\mathrm{Kn}$. wiederum bedeutet gleichzeitige Bengung in diesen beiden Gelenken.

Tabelle I.

Reaktion auf Temperaturreize nach Exstirpation der Extremitätenregion rechts. Hintere Extremität.

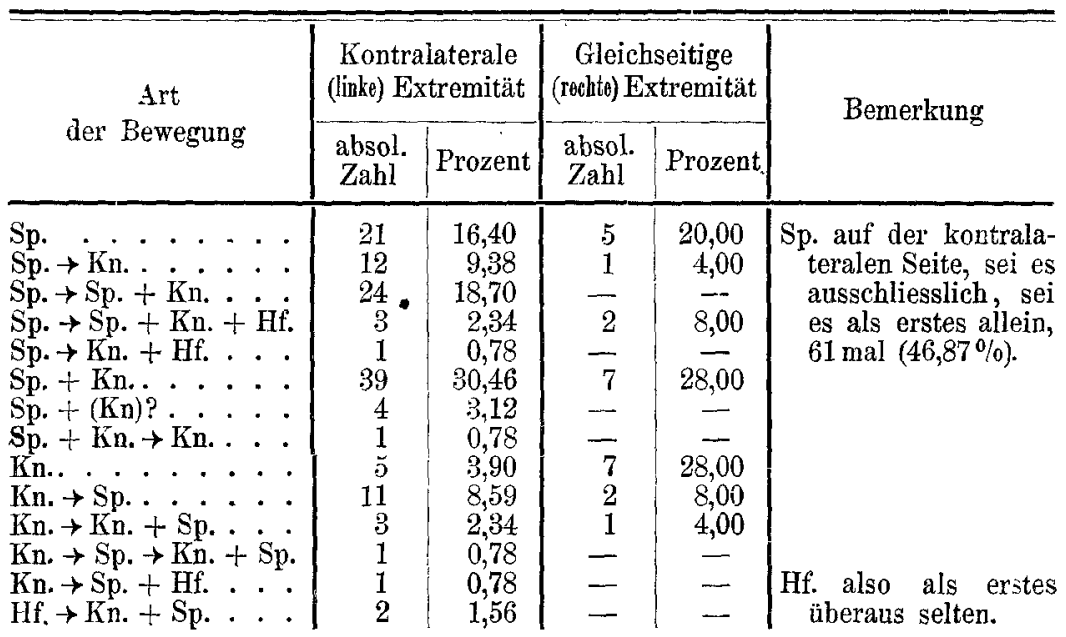

Tabelle II.

Reaktion auf Temperaturreize nach Exstirpation der Extremitätenregion rechts. Vordere Extremität.

\begin{tabular}{|c|c|c|c|c|c|}
\hline \multirow{2}{*}{$\begin{array}{c}\text { Art } \\
\text { der Bewegung }\end{array}$} & \multicolumn{2}{|c|}{$\begin{array}{c}\text { Kontralaterale } \\
\text { (linke) Extremität }\end{array}$} & \multicolumn{2}{|c|}{$\begin{array}{c}\text { Gleichseitige } \\
\text { (recbte) Extremität }\end{array}$} & \multirow{2}{*}{ Bemerkung } \\
\hline & $\begin{array}{c}\text { absol. } \\
\text { Zahl }\end{array}$ & Prozent & $\begin{array}{l}\text { absol. } \\
\text { Zahl }\end{array}$ & Prozent & \\
\hline 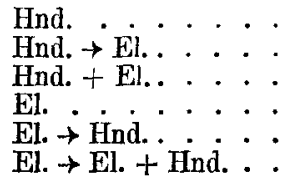 & $\begin{array}{r}2 \\
3 \\
28 \\
11 \\
6 \\
2\end{array}$ & $\begin{array}{r}3,84 \\
5,77 \\
53,84 \\
21,15 \\
11,54 \\
3,84\end{array}$ & $\frac{-}{\overline{4}}$ & $\begin{array}{l}- \\
\overline{50,00} \\
37,50 \\
\overline{12,75}\end{array}$ & \\
\hline
\end{tabular}




\section{Tabelle III.}

In Morphium-(teilweise Chloralhydrat und Chloroform)narkose; Reaktion auf Temperaturreize (nach Exstirpation der Extremitätenregion rechts). Hintere Extremität.

\begin{tabular}{|c|c|c|c|c|c|}
\hline \multirow{2}{*}{$\begin{array}{c}\text { Art } \\
\text { der Bewregung }\end{array}$} & \multicolumn{2}{|c|}{$\begin{array}{c}\text { Kontralaterale } \\
\text { (linke) Extremität }\end{array}$} & \multicolumn{2}{|c|}{$\begin{array}{c}\text { Gleichseitige } \\
\text { (redlite) Extremität }\end{array}$} & \multirow{2}{*}{ Bemerkung } \\
\hline & $\begin{array}{l}\text { absol. } \\
\text { Zahl }\end{array}$ & Prozent & $\begin{array}{l}\text { absol. } \\
\text { Zahl }\end{array}$ & Prozent & \\
\hline 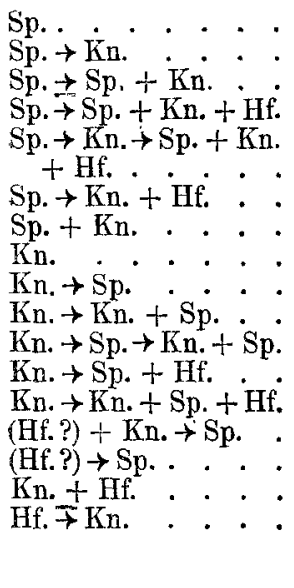 & $\begin{array}{r}17 \\
8 \\
17 \\
5 \\
2 \\
4 \\
4 \\
14 \\
15 \\
18 \\
5 \\
1 \\
1 \\
2 \\
3 \\
2\end{array}$ & $\begin{array}{r}14,91 \\
7,01 \\
14,91 \\
4,38 \\
1,75 \\
\\
3,50 \\
12,28 \\
13,16 \\
15,79 \\
4,38 \\
0,877 \\
0,- \\
1,777 \\
2,62 \\
1,75\end{array}$ & $\begin{array}{r}10 \\
-\frac{1}{2} \\
- \\
- \\
- \\
7 \\
8 \\
2 \\
- \\
\\
- \\
- \\
-\end{array}$ & $\begin{array}{c}26,31 \\
15,78 \\
\overline{5,26} \\
- \\
- \\
\overline{-} \\
18,42 \\
21,04 \\
5,26 \\
2,68 \\
\overline{5,26} \\
= \\
= \\
-\end{array}$ & $\begin{array}{l}\text { Sp. anf der kontralatera- } \\
\text { len Seite, sei es aus- } \\
\text { schliesslich sei es als } \\
\text { erstes alliein, 53 mal } \\
(46,4 \% 0) ; \text { gleichs. } 18 \mathrm{mal} \\
(42,1 \%) \text {. }\end{array}$ \\
\hline
\end{tabular}

Tabelle IV.

In Morphium-(teilweise Chloralhydrat und Chloroform)narkose; Reaktion auf Temperaturreize (nach Exstirpation der Extremitätenregion rechts). Vordere Extremität.

\begin{tabular}{|c|c|c|c|c|c|}
\hline \multirow{2}{*}{$\begin{array}{c}\text { Art } \\
\text { der Bewegung }\end{array}$} & \multicolumn{2}{|c|}{$\begin{array}{c}\text { Kontralaterale } \\
\text { (linke) Extremität }\end{array}$} & \multicolumn{2}{|c|}{$\begin{array}{c}\text { Gleichseitige } \\
\text { (rechte) Extremität }\end{array}$} & \multirow{2}{*}{ Bemerkung } \\
\hline & $\begin{array}{l}\text { absol. } \\
\text { Zahl }\end{array}$ & Prozent & $\begin{array}{l}\text { absol. } \\
\text { Zahl }\end{array}$ & Prozent & \\
\hline 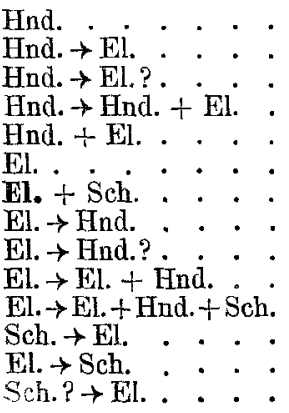 & $\begin{array}{r}4 \\
7 \\
2 \\
-14 \\
27 \\
1 \\
3 \\
1 \\
2 \\
1 \\
1 \\
\frac{1}{1}\end{array}$ & $\begin{array}{r}6,25 \\
10,90 \\
3,12 \\
- \\
21,81 \\
42,18 \\
1,56 \\
4,68 \\
1,56 \\
3,12 \\
1,56 \\
1,56 \\
\overline{1,56}\end{array}$ & $\begin{array}{r}\overrightarrow{3} \\
\frac{2}{5} \\
10 \\
\frac{2}{2} \\
\frac{1}{1} \\
\frac{1}{1} \\
-\end{array}$ & $\begin{array}{r}\overline{12} \\
\overline{8} \\
20 \\
\frac{40}{8} \\
\frac{4}{4} \\
\frac{4}{-}\end{array}$ & \\
\hline
\end{tabular}


Zur Lehre Munk's über Beginn und Reihenfolge in der Ausbreitung etc. 51

Tabelle V.

Reaktion auf Temperaturreize nach Rückenmarksdurchschneidung. Hintere Extremitäten.

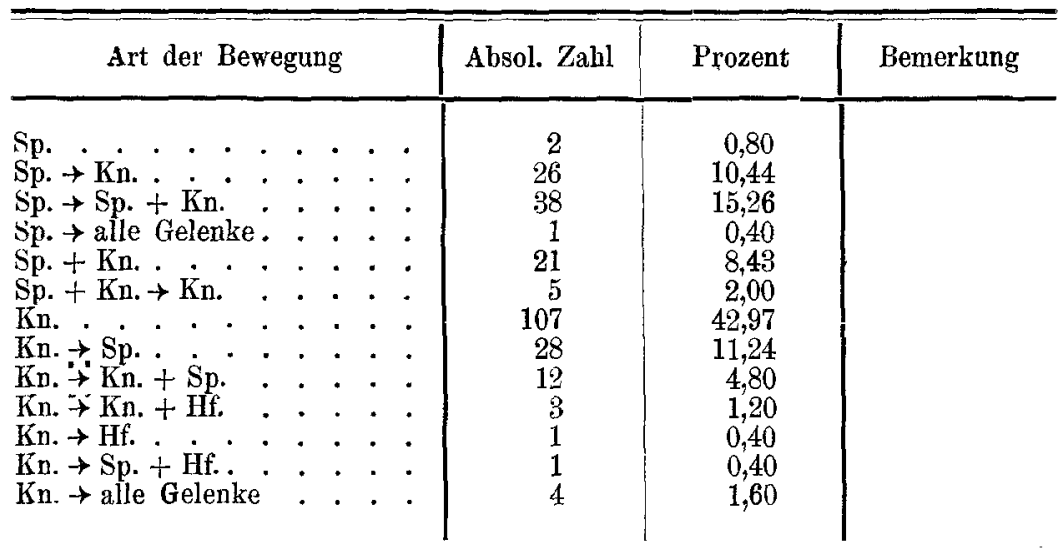

Diese Tabellen zeigen augenfällig, dass nicht nur nach Exstirpation der Extremitätenregion, sondern selbst nach Rückenmarksdurchschneidung Einzelbewegungen, beschränkt auf das unterste Gelenk (Sprunggelenk, ev. Handgelerik), vorkommen, sogar in einer recht beträchtlichen Prozentzahl. Weiter ist ersichtlich, dass die Reihenfolge in der Beteiligung verschiedener Gelenke derselben Extremität absolut nicht den Anforderungen Munk's entspricht. Wir sehen nämlich häufig, dass auch in der der Verbindung mit der Extremitätenregion der Hirnrinde beraubten Extremität gar nicht selten die Beteiligung der Gelenke eine von unten (Sprung-Handgelenk) nach oben (Knie-Ellbogengelenk) fortschreitende ist. Eine Bewegung zuerst betreffend nur das oberste Glied (Hüft-Schultergelenk) einer Extremität bei einer in Intervallen sich abspielenden Reihenfolge von Bewegungen mehrerer Glieder ist überhaupt eine grosse Seltenheit; eine Kontraktion wiederum bloss im obersten Gelenk (ohne Beteiligung der unteren Gelenke) ist überbaupt nicht beobachtet worden. Dies stimmt wiederum mit den zitierten Angaben Munk's nicht überein. Was sich allerdings zeigte und auf den ersten Anblick eine zum wenigsten teilweise Bestätigung der Angaben Munk's zu liefern scheint, ist die grosse Häufigkeit mit der die Zurückziehung im Kniegelenke resp. Ellenbogengelenke geschab, sei es ausschliesslich, sei es als Beginn von sukzessiv aufeinanderfolgenden Kontraktionen.

Jedoch jst die grosse Anspruchsfähigkeit der Zentra für das Knie- resp. Ellenbogengelenk (wie wir im nachfolgenden dartun 
werden) keine ausschliessliche Eigenschaft der vom zerebralen Einfluss ausgeschalteten Extremität und kann daher dafür die eingangs angegebene Auffassung Munk's nicht als Erklärung dienen.

Wir konstatierten nämlich, dass ein plötzlicher stärkerer Druck an den Zehen oder auch an der Pfote bei mögliehster Vermeidung eines eigentlichen taktilen Reizes sowohl an der pathologischen als auch an der intakten Extremität zunächst eineBeugung im Kniegelenke hervorruft und erst hierauf eventuell auch in den anderen Gelenken. Um möglichst einen taktilen Reiz auszuschalten, umbüllten wir die Zehen und den unteren Abschnitt der Pfote bei vollständiger Freilassung der Beweglichkeit im Sprunggelenke mit einer dicken Schicht Watte und einer Kalikotbinde und drückten hierauf plötzlich mit einiger Intensität die Zehen resp. den umhüllten Teil der Pfote. Der Erfolg einer derartigen mechanischen Reizung ist nun, wie bereits erwähnt, bei der weitaus überwiegenden Zahl der untersuchten Tiere ganz derselbe, ob die Extremität ihrer Verbindung mit der Extremitätenregion beraubt ist, oder nicht.

Beim Hund scheint überhaupt das Knie- resp. Ellenbogengelenk ein bei lokomotorischen Bewegungen des Tieres augenscheinlich am meisten aktives Gelenk - beim reflektoriseh hervorgerufenen Verkürzungsvorgang der Extremität am leichtesten beteiligt und dessen Rückenmarkszentrum reflektorisch am leichtesten beansprucht $\mathrm{zu}$ sein.

Auch Reizung mittels faradischen Stromes durch Einstechen von Elektroden in die Haut der Pfote, gewöhnlich an der Dorsalseite (siehe Tabelle VI, VII, VIII), verursachte bei Anwendung von minimalen Stromstärken zunächst (I. Stadium) an den hinteren Extremitäten bei weitem überwiegend eine reflektorische Beugung im Kniegelenk, obne wesentlichen Unterschied, ob die der Exstirpation gleichseitige oder kontralaterale Extremität gereizt wurde. Auch an der vorderen Extremität überwiegt bei derartiger faradischer Reizung mit noch nicht intensiver Stromstärke jedenfalls eine Beugung im Ellenbogengelenk. Wurde hierauf mit stärkeren Strömen (II. Stadium) neuerdings gereizt, so erfolgte ausser einer erneuten Beugung im Knie- resp. Ellenbogengelenk noch eine solche in einem unteren eventuell in einem oberen Gelenk (manchmal in allen); endlich erfolgte bei sebr starken Strömen in der Regel eine vollständige Verkürzung der Extremität in allen Gelenken (III. Stadium). 


\section{Tabelle VI ${ }^{1}$ ).}

Reihenfolge der bei steigender Stromstärke beteiligten Gelenke. Exstirpation der rechten Extremitätenregion. Hintere Extremität.

\begin{tabular}{|c|c|c|c|}
\hline Art der Bewegung & $\begin{array}{l}\text { Kontra- } \\
\text { laterale Ex- } \\
\text { tremität }\end{array}$ & $\begin{array}{c}\text { Gleichseitige } \\
\text { Extremität }\end{array}$ & $\begin{array}{l}\text { In Narkose, } \\
\text { beide hintere } \\
\text { Extremitäten }\end{array}$ \\
\hline 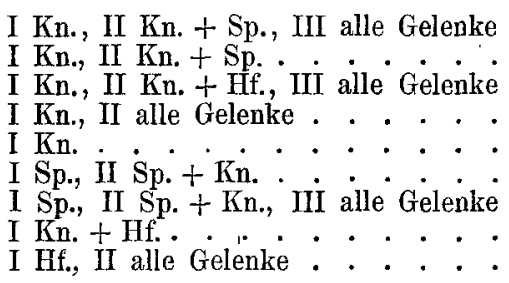 & $\begin{array}{l}3 \\
1 \\
1 \\
1 \\
1 \\
- \\
-\end{array}$ & $\begin{array}{l}1 \\
1 \\
2 \\
1 \\
\\
- \\
1 \\
1\end{array}$ & $\begin{array}{l}5 \\
7 \\
3 \\
5 \\
1 \\
\frac{1}{1} \\
\frac{-}{-}\end{array}$ \\
\hline
\end{tabular}

\section{Tabelle VII.}

Reihenfolge der bei steigender Stromstärke beteiligten Gelenke. Exstirpation der rechten Extremitätenregion. Vordere Extremität.

\begin{tabular}{|c|c|c|c|}
\hline Art der Bewegung & $\begin{array}{c}\text { Kontra- } \\
\text { laterale } \\
\text { vordere Ex- } \\
\text { tremität }\end{array}$ & $\begin{array}{c}\text { Gleichseitige } \\
\text { vordere } \\
\text { Extremität }\end{array}$ & $\begin{array}{l}\text { In Narkose } \\
\text { beide } \\
\text { vordere Ex- } \\
\text { tremitäten }\end{array}$ \\
\hline 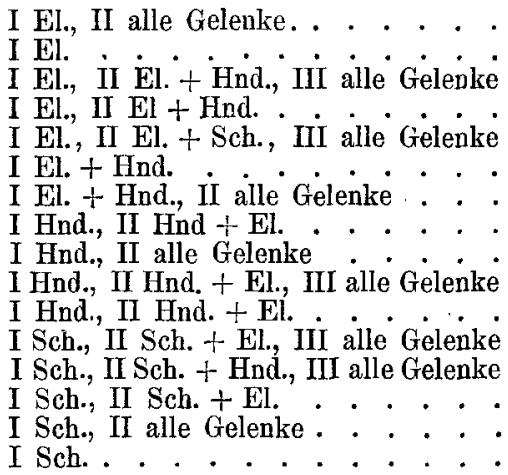 & $\begin{array}{l}\frac{2}{1} \\
\frac{1}{-} \\
\overline{1} \\
\overline{-} \\
- \\
\overline{-} \\
1 \\
1 \\
1 \\
1\end{array}$ & $\begin{array}{l}- \\
- \\
- \\
\frac{1}{2} \\
\frac{2}{-} \\
\frac{1}{-} \\
-\end{array}$ & $\begin{array}{l}2 \\
1 \\
6 \\
2 \\
2 \\
1 \\
1 \\
\frac{1}{3} \\
2 \\
2 \\
\\
2 \\
1\end{array}$ \\
\hline
\end{tabular}

1) Die Abkürzungen in Tabelle VI-VIII bezeichnen dieselben Gelenke wie in den vorigen Tabellen. Ferner bedeutet I die bei relativ schwächstem Strom erhaltene Bewegung, II die bei stärkerem Strome noch ein benachbartes Gelenk (resp. noch zwei benachbarte Gelenke) betreffende Bewegung, III die bei relativ stärkstem Strom resultierte Bewegung. In manchen Fällen war der Effekt der Reizung bei maximaler Stromstärke nicht anders als im Stadium II, manchmal sogar wie im Stadium I, und dann ist in den entsprechenden Tabellen nur das Resultat von Stadien I und II eventuell auch nur von Stadium I angeführt. 


\section{Tabelle VIII.}

Reihenfolge der bei steigender Stromstärke beteiligten Gelenke. Nach der Durchschneidung des Rückenmarkes.

\begin{tabular}{l|r}
\hline Art der Bewegung & \\
\hline & \\
\hline I Kn., II Kn. + Sp., III alle Gelenke . . . . . & 10 \\
I Kn., II alle Gelenke . & 5 \\
I Kn., II Kn. + Sp. oder Kn. + Hf., III alle Gelenke & 1 \\
I Kn., II Kn. + Hf., III alle Gelenke . • . . & 4
\end{tabular}

Beachtenswert ist nun jedenfalls, dass im II. Stadium das Weiterschreiten (von Knie-und Ellenbogengelenk) in der Beteiligung der Gelenke an der Verkürzung der Extremität eine ebenso häufige in distaler (Sprung-Handgelenk) wie in proximaler (Hüft-Schultergelenk) Richtung ist.

Unsere Versuchsergebnisse können wir in folgenden Sätzen zusammenfassen :

1. Sonderbewegungen auch im untersten Gelenke der Extremitäten als reflektorische Bewegung kommen nicht nur nach Exstirpation der Extremitätenregion der Hirnrinde, sondern selbst nach Rückenmarksdurchschneidung gar nicht selten vor, und zwar sowohl bei einem typischen Hautreflex (unserem Plantarreflex) wie auch bei Reflexen nach thermischer Reizung.

2. Nicht das oberste Gelenk ist es, in welchem nach Exstirpation der Extremitätenregion (oder nach Rückenmarksdurchschneidung) bei Reizung an der Pfote irgendwie häufig eine reflektorische Bewegungsreaktion zum Vorschein kommt, sondern das Knie- resp. Ellenbogengelenk. Die mehr oder weniger grössere Anspruchsfähigkeit dieser letzeren Gelenke jst auch schon am intakten Tiere (bei faradischer Reizung, eventuell bei rapidem, starkem Druck) vorhanden.

3. Die Reihenfolge in den reflektorisch sukzessiv zur Aktion gelangenden Gelenken nach Ausschaltung der kortikalen Verbindung, selbst nach Rückenmarksdurchschneidung, entspricht meist nicht den Anforderungen Munk's. Wir sehen nämlich bei Andauer der thermischen Reizung auf eine Dorsalflexion im Sprunggelenke sehr häufig eine Beugung im Kniegelenke folgen, also in diesen Fällen entgegen Munk ein Fortschreiten in der Beteiligung der Glieder in der Richtung von unten nach oben. Ebenfalls stimmt nicht überein 
mit dem Schema der Reihenfolge Munk's der Erfolg elektrischer Reizung mit anwachsenden Strömen.

4. Die fast willkürliche Annahme Munk's, wonach die sensiblen Nervenfasern des Rückenmarks, sei es als solche, sei es vermittels Zellen, nur mit den Rückenmarkszentren für die obersten Glieder der Extremität in direkter Verbindung stehen und erst mittelbar durch diese Zentren auch die Rückenmarkszentren für die unteren Glieder in Erregung, setzen sollten, müssen wir, wie die Ergebnisse sub 1, 2 und 3 ganz überzeugend dartun, als ganz unbegründet betrachten. Es liegt auch weder physiologisch noch anatomisch irgendein Grund vor für eine derartige Annahme einer Beschränkung der zentripetalen direkten Zuleitung der Erregung ausschliesslich an ein, ein einziges Gelenk innervierendes, Rückenmarkszentrum. Die unzweifelhaft leichtere, aber keinesfalls ausschliessliche Anspruchsfähigkeit des Zentrums für eine (reflektorische) Beugung im Knie- resp. Ellenbogengelenk ist nicht Folge etwaiger morphologischer Verhältnisse, sondern vielmehr als Ausdruck einer physiologisehen funktionellen Bahnung zu betrachten.

5. Das weitere Postulat Munk's, dass für die sog. Prinzipalzentra und für die Extremitätenregion der Hirnrinde gesonderte Leitungsbahnen zum Rückenmark von verschiedener Verbindungsweise mit den einzelnen motorischen Rückenmarkszentren vorhanden sein müssten, zeigt sich angesichts obiger Auseinandersetzungen als überflüssig. Ausserdem mag auch eine und dieselbe (zerebrospinale) Leitungsbahn bei intakter Verbindung mit der Extremitätenregion vom Willen fortwährend korrigierte und sichtlich beeinflusste $\mathrm{Be}$ wegungen erzeugen, während dieselben nach Ausschluss der Hirnrinde mehr den Charakter von Reflexbewegungen erhalten.

\section{Anhang, betreffend die Haltung der Extremitäten nach einseitiger Exstirpation der Extremitätenregion and die Einwirkung des Morphins anf diese Haltung.}

Bei Tieren, denen die Extremitätenregion der Hirnrinde einseitig exstirpiert wurde, zeigt sich wie bekannt, wenn dieselben vertikal hängend gehalten werden, nicht selten eine mit Rigidität verbundene Streckung der kontralateralen Extremitäten. Dasselbe Phänomen kommt beim Hunde auch zum Vorschein, wenn das Tier horizontal in eine Hängematte, aus der die Extremitäten frei nach unten herausragen, sich befindet. Die decerebrate rigidity der englischen Autoren 
(Sherrington) tritt beim Hunde auch in den erwähnten. Lagen nicht immer auf, ist aber jedenfalls zeitweilig sehr ausgeprägt vorhanden. Man sieht dann eventuell die hintere kontralaterale Extremität im Hüftgelenk nicht selten nach hinten gerichtet, im Kniegelenk gestreckt, auch im Sprunggelenke manchmal eine ausgesprochene tonische Plantarflexion. Die-vordere kontralaterale Extremität zeigt wiederum häufig Streckung im Ellbogen- und Handgelenk mit eventueller Hyperextension der Finger. Ein Versuch, diese extensorische Haltung durch passive Beugung aufzuheben, trifft auf geringeren oder stärkeren Widerstand ${ }^{1}$ ). Der Kontrast $z$ wischen der Haltung der kontralateralen (extendierten) und der gleichseitigen (flektierten) Extremität ist dann nicht selten ein recht bedeutender, sogar auffälliger und betrifft sei es vordere und hintere, sei es nur die hinteren oder vorderen Extremitäten.

Sowohl die Rigidität als auch die angegebene Streckung der kontralateralen Extremitäten beim Hunde bilden nicht nur keine konstante Erscheinung, sondern wechseln sogar im Grade und Intensität ziemlich rasch. Eine zunächst in mässiger Extension gehaltene kontralaterale Extremität kann nach einer Weile denselben Grad der Beugung zeigen wie die gleichseitige Extremität. Jedoch trug es sich in allen unseren Versuchen kein einziges Mal, wenn das Tier im wachen Zustand war, zu, dass die Bengung der kontralateralen Extremitäten irgendwie bedeutender wäre als an der gleichseitigen.

Es war uns daher sehr auffallend, als wir bei einem Hunde mit einseitig exstirpierter Extremitätenregion, dem wir zu anderen Zwecken während der Beobachtungszeit salzsaures Morphin unter die Haut injiziert haben, ein ganz abweichendes Verhalten konstatierten.

Es zeigte sich nämlich während der Morphiumnarkose die kontralaterale früher auffällig stark extendierte hintere Extremität bedeutend stärker in allen Gelenken gebeugt als auf der gleichen Seite (siehe Fig. 1 und 2). In weiteren Untersuchungen sowohl an

1) Manchmal ist allerdings eine gewisse Streckung der kontralateralen Extremitäten ohne Rigidität vorhanden. Die Streckung der kontralateralen Extremität bei den angeführten Positionen ist, wenn ohne Rigor, wahrscheinlich nur der Ausdruck eines verringerten Tonus infolge Wegfalls des kortikalen Einflusses auf den Beugetonus. Dagegen ist die mit Rigidität einhergehende Streckung der kontralateralen Extremität einzig als eine reflektorische aktive Streckung anzusehen (in letzterer Beziehung vergleiche Sherrington, The integrative action of the nervous system. 1906). 
Zur Lehre Munk's über Beginn und Reihenfolge in der Ausbreitung etc. 57

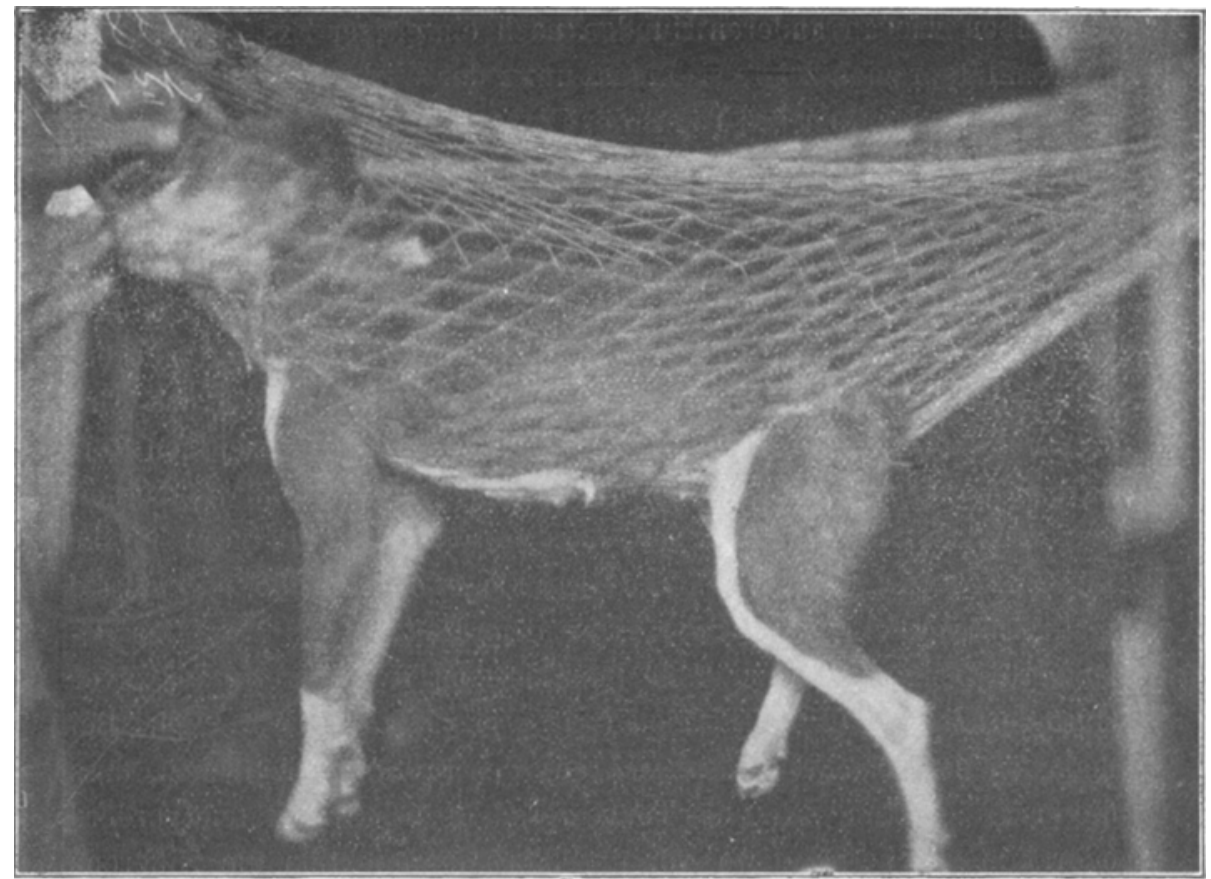

Fig. 1. Haltung der (besonders hinteren) Extremitäten vor der Narkose.

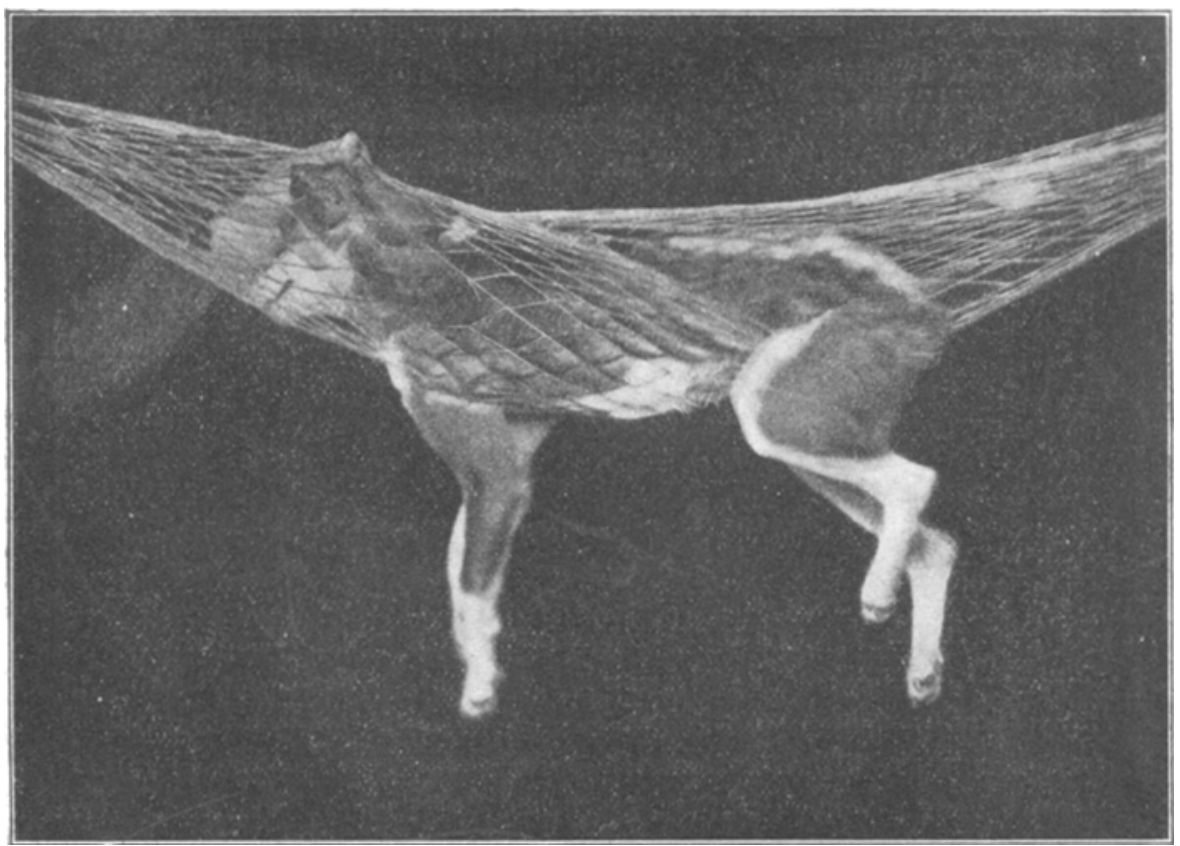

Fig. 2. Umkehr der Haltung bei demselben Hunde während der Morphiumnarkose. 
demselben wie àn anderen Hunden nach einseitiger Exstirpation der Extremitätenregion zeigte sich, dass unter der Wirkung der Morphiumnarkose eine Umkehr des Tonus der Extremitäten in oben angegebener Weise, das ist eine stärkere Flexion gerade der kontralateralen (früher stärker gestreckten) Extremität nicht selten ist. Wir beobachteten dies deutlich bei fünf (von 10) operierten Hunden, dazu noch bei einem Tiere in minder ausgesprochenem Grade. Die derartige Umkehrung des Tonus betraf manchmal beide Extremitätenpaare, manchmal nur die hinteren oder vorderen. Wovon das Eintreten oder das Ausbleiben einer Änderung im beiderseitigen Verhältnis des Tonus abhängig ist, konnten wir nicht mit Sicherheit eruieren. Es scheint allerdings, dass es sich hier um eine reziproke Innervationserscheinung im Sinne S h er r in g t on's handle, und müsste man sich vorstellen, dass der unter dem Einfluss des Morphins gesteigerte Beugungstonus gerade da intensiver sich äussere, wo zuvor statt der Flexion eine Extension (mit oder ohne Rigor) bestand. 'Bei manchen Versuchstieren lässt sich aus den Protokollen ersehen, dass die erwähnte Umkehrung des Tonus gerade jene Extremitäten (vordere oder hintere) betrifft, wo vor der Morphiuminjektion die Differenz in der beiderseitigen Haltung eine ausgesprochenere war. Doch ist. es absolut nicht durchgreifend der Fall, und gibt es jedenfalls keinen strengen Parallelismus zwischen der gegenseitigen Haltung vor und nach der Injektion von Morphin. Auch sei noch bemerkt, dass unter dem Einfluss von Morphin bezüglich der vorderen Extremitäten nicht immer eine Steigerung im Tonus der Beuger, sondern manchmal gerade eine Streckung der vorderen Extremitäten beobachtet wurde. 